\title{
Fast Computations in Cortical Ensembles Require Rapid Initiation of Action Potentials
}

\author{
Vladimir Ilin, ${ }^{1 \star}$ Aleksey Malyshev, ${ }^{1,2 \star}$ Fred Wolf, ${ }^{3}$ and Maxim Volgushev ${ }^{1,2}$ \\ ${ }^{1}$ Department of Psychology, University of Connecticut, Storrs, Connecticut 06269, ${ }^{2}$ Institute of Higher Nervous Activity and Neurophysiology, Russian \\ Academy of Sciences, Moscow 117485, Russia, and ${ }^{3}$ Max Planck Institute for Dynamics and Self-Organization and Bernstein Center for Computational \\ Neuroscience, D-37077 Göttingen, Germany
}

The abilities of neuronal populations to encode rapidly varying stimuli and respond quickly to abrupt input changes are crucial for basic neuronal computations, such as coincidence detection, grouping by synchrony, and spike-timing-dependent plasticity, as well as for the processing speed of neuronal networks. Theoretical analyses have linked these abilities to the fast-onset dynamics of action potentials (APs). Using a combination of whole-cell recordings from rat neocortical neurons and computer simulations, we provide the first experimental evidence for this conjecture and prove its validity for the case of distal AP initiation in the axon initial segment (AIS), typical for cortical neurons. Neocortical neurons with fast-onset APs in the soma can phase-lock their population firing to signal frequencies up to $\sim 300-400 \mathrm{~Hz}$ and respond within $1-2$ ms to subtle changes of input current. The ability to encode high frequencies and response speed were dramatically reduced when AP onset was slowed by experimental manipulations or was intrinsically slow due to immature AP generation mechanisms. Multicompartment conductancebased models reproducing the initiation of spikes in the AIS could encode high frequencies only if AP onset was fast at the initiation site (e.g., attributable to cooperative gating of a fraction of sodium channels) but not when fast onset of somatic AP was produced solely by backpropagation. We conclude that fast-onset dynamics is a genuine property of cortical AP generators. It enables fast computations in cortical circuits that are rich in recurrent connections both within each region and across the hierarchy of areas.

\section{Introduction}

Cortical neurons can encode rapidly varying signals by phaselocking their firing to high-frequency fluctuations (Köndgen et al., 2008; Boucsein et al., 2009; Higgs and Spain, 2009; Tchumatchenko et al., 2011) and respond very quickly, on a millisecond timescale, to changes of input current (Tchumatchenko et al., 2011). Theoretical analyses identify these properties as characteristics of action potential (AP) generators with fast-onset dynamics (Brunel et al., 2001; Fourcaud-Trocmé et al., 2003; Naundorf et al., 2005; Wei and Wolf, 2011). Populations of model neurons with instant AP generation, such as leaky integrate-and-fire models (Knight 1972; Tuckwell, 1998), can encode in their firing any frequency present in membrane potential fluctuations. In contrast, populations of conductance-based model neurons with finite AP onset dynamics cannot encode

Received Feb. 16, 2012; revised 0ct. 31, 2012; accepted Nov. 9, 2012.

Author contributions: A.M., F.W., and M.V. designed research; V.I., A.M., and M.V. performed research; V.I. contributed unpublished reagents/analytic tools; V.I., A.M., and M.V. analyzed data; V.I., A.M., F.W., and M.V. wrote the paper.

This work was supported by grants from the German-Israeli Foundation (F.W., M.V.), Max Planck Society (F.W.), Russian Foundation of Basic Research (A.M.), and University of Connecticut startup funds (M.V.). We thank S. Volgushev for help with data processing, M. Chistyakova, J. Chrobak, M. Escabi, I. Fleidervish, M. Gutnik, A. Neef, J. Salamon, H. Swadlow, T. Tchumatchenko, and W. Wei for critical discussions and comments on this manuscript, and J. Chrobak for improving the English.

*V.I. and A.M. contributed equally to this work.

The authors declare no competing financial interests.

Correspondence should be addressed to Maxim Volgushev, University of Connecticut, Department of Psychology, 406 Babbidge Road, Unit 1020, Storrs, CT 06269-1020. E-mail: maxim.volgushev@uconn.edu.

DOI:10.1523/JNEUROSCI.0771-12.2013

Copyright $\odot 2013$ the authors $\quad 0270-6474 / 13 / 332281-12 \$ 15.00 / 0$ frequencies several-fold higher than the mean firing rate, the cutoff frequency depending on AP onset dynamics (FourcaudTrocmé et al., 2003; Wei and Wolf, 2011). However, this relationship has not been tested experimentally. It also remains unclear whether theoretical conclusions derived from analysis of single-compartment models (Brunel et al., 2001; FourcaudTrocmé et al., 2003; Naundorf et al., 2005; Wei and Wolf, 2011) hold for real neocortical neurons in which APs are initiated in the axon, 30-40 $\mu \mathrm{m}$ away from the soma (Stuart and Sakmann, 1994; Stuart et al., 1997; Palmer and Stuart, 2006). The ability to encode high-frequency signals was suggested as a functional test to distinguish whether fast onset of APs in the soma represents a genuine property of cortical AP generators (Naundorf et al., 2006, 2007) or is an epiphenomenon produced solely by backpropagation ( $\mathrm{Yu}$ et al., 2008). In the latter scenario, APs have a slow onset at the initiation site but become fast while invading the soma.

Here we set out to bridge this gap between theory and experiment and to test the above predictions using a combination of whole-cell recordings from neurons in rat neocortex and computer simulations. We show that neocortical neurons with fastonset APs in the soma can phase-lock their population firing to signal frequencies up to $\sim 300-400 \mathrm{~Hz}$ and respond within $1-2$ $\mathrm{ms}$ to subtle changes of input current. The ability to encode high frequencies and response speed were dramatically reduced by experimental manipulations that slowed AP onset dynamics and in immature neurons. In multicompartmental conductancebased models that reproduced spatial pattern of AP initiation in the axon initial segment (AIS) and propagation down the axon 
and back to the soma and dendrites, encoding depended on AP onset dynamics at the initiation site. Rapidly changing signals could be encoded by models with fast AP initiation, achieved by introducing threshold activation or cooperative gating to a fraction of sodium channels in the AIS but not by models with a slow, Hodgkin-Huxley-type AP initiation, even when tuned to produce APs with fast onset in the soma. We conclude that fast-onset dynamics is not an epiphenomenon that can be explained solely by backpropagation. Rather, fast-onset dynamics is a genuine property of cortical AP generators that enables fast computations in cortical circuits and allows to reduce dramatically the size of neuronal ensembles and required connectivity.

\section{Materials and Methods}

All experimental procedures used in this study conformed to National Institutes of Health regulations. Experimental protocols were approved by the respective local animal welfare committees (Bezirksregierung Arnsberg, Germany, and Institutional Animal Care and Use Committee of University of Connecticut). Wistar rats (Charles River or Harlan) of either sex of two age groups were used. P17-P30 (mean of P23.5, median of P23) rats were used in all experimental series except for experiments on encoding in neurons from young animals, in which P9-P13 (mean of $\mathrm{P} 11.8$, median of $\mathrm{P} 12$ ) rats were used. In vitro intracellular recordings were made in slices of rat visual cortex using slice preparation and recording techniques as in our previous work (Volgushev et al., 2000; Tchumatchenko et al., 2011). Rats were anesthetized with isoflurane (Baxter) and decapitated, and the brain was rapidly removed. One hemisphere was mounted onto an agar block, and $350-\mu \mathrm{m}$-thick coronal slices containing area 17 and area 18 of the visual cortex were cut with a vibratome (Leica VT1000S) in ice-cooled oxygenated solution. Slices containing the visual cortex were selected for recording based on a characteristic triangular shape of underlying white matter, corresponding to coronal slices between approximately $-5.3 \mathrm{~mm}$ and approximately $-6.8 \mathrm{~mm}$ from bregma in adult male rat brain (Paxinos and Watson, 1997). After cutting, the slices were placed into an incubator in which they recovered for at least $1 \mathrm{~h}$ at room temperature. For recording, one slice was transferred into a recording chamber of submerged type. The solution used during the preparation of the slices had the same ionic composition as the perfusion/extracellular solution. It contained the following (in mM): $125 \mathrm{NaCl}, 2.5 \mathrm{KCl}, 2 \mathrm{CaCl}_{2}, 1 \mathrm{MgCl}_{2}, 1.25 \mathrm{NaH}_{2} \mathrm{PO}_{4}, 25$ $\mathrm{NaHCO}_{3}$, and 25 D-glucose (bubbled with $95 \% \mathrm{O}_{2}$ and $5 \% \mathrm{CO}_{2}$ ). In some experiments, synaptic transmission was blocked by adding $25 \mu \mathrm{M}$ aminophosphonovalerate (APV), $5 \mu \mathrm{M}$ 6,7-dinitroquinoxaline-2,3dione (DNQX), and $80 \mu \mathrm{M}$ picrotoxin to the extracellular solution. In experiments with reduced extracellular $\left[\mathrm{Na}^{+}\right], \mathrm{NaCl}$ was partially substituted by choline chloride, so that $\left[\mathrm{Na}^{+}\right]$in the final solution was 56.25 $\mathrm{mm}$ compared with $151.25 \mathrm{~mm}$ in control extracellular solution. All chemicals were obtained from Sigma-Aldrich.

Recordings. Recordings were made with the slices in submerged conditions at $28-32^{\circ} \mathrm{C}$. Temperature in the recording chamber was monitored with a thermocouple positioned close to the slice, $2-3 \mathrm{~mm}$ from the recording site. Whole-cell recordings using patch electrodes were made from layer $2 / 3$ pyramidal neurons in the visual cortex, selected under visual control using Nomarski optics and infrared video microscopy. All recordings were made from the soma, so all parameters of APs in neurons in slices (such as onset dynamics, amplitude, etc.) were measured in the soma. The patch electrodes were filled with K-gluconate-based solution (in mM: $130 \mathrm{~K}$-gluconate, $20 \mathrm{KCl}, 4 \mathrm{Mg}$-ATP, $0.3 \mathrm{Na}_{2}$-GTP, $10 \mathrm{Na}$ phosphocreatine, and 10 HEPES) and had a resistance of $4-6 \mathrm{M} \Omega$. Recordings were performed using the bridge mode of Axoclamp-2A (Molecular Devices) or Dagan BVC-700A (Dagan Corporation) amplifier. After amplification and low-pass filtering at $10 \mathrm{kHz}$, data were digitized at $20 \mathrm{kHz}$ and fed into a computer (Digidata 1440A interface and pClamp software; Molecular Devices).

Fluctuating current, $I(t)$, for injection into a neuron was synthetized using the following equation:

$$
I(t)=I_{0}+\sigma_{\text {noise }} \eta(t),
$$

where $\eta(t)$ is an Ornstein-Uhlenbeck process with zero mean, unit variance, and correlation time $\tau_{\text {noise }}=5 \mathrm{~ms}$, and $\sigma_{\text {noise }}$ is the SD of the resulting background current noise. This noise component $\sigma_{\text {noise }} \eta(t)$ mimics the effect produced in the soma by numerous balanced excitatory and inhibitory synaptic inputs (Destexhe et al., 2003). A direct current $\left(I_{0}\right)$ was added if necessary to maintain desired firing rate of 3-6 Hz. Membrane potential fluctuations produced by the injected current were similar to those recorded in neocortical neurons in vivo (Azouz and Gray, 2000; Destexhe et al., 2003; Volgushev et al., 2006). Current injections lasted $46 \mathrm{~s}$ and were separated by a recovery period of $60-100$ s. For experiments described in Figures 2 and 5, a weak sine-wave signal was added to the fluctuating noise. For experiments described in Figure 7, small current steps $(20 \mathrm{pA})$ were added to the fluctuating noise.

Computer simulations. Computer simulations were performed in NEURON environment (http://www.neuron.yale.edu/neuron/, version 7.1; Hines et al., 2004; Carneval and Hines, 2006) using recently published models (Baranauskas et al., 2010; Fleidervish et al., 2010). In the model by Fleidervish et al. (2010) (available online at ModelDB database, http://senselab.med.yale.edu/senselab/modeldb), virtual temperature was changed from $6.3^{\circ} \mathrm{C}$ to $32^{\circ} \mathrm{C}$, and sodium conductance $(g \mathrm{Na}$ ) was increased by 2.5 times in all compartments to achieve generation of multiple spikes in response to injected fluctuating current, according to recommendation of the authors (Ilya Fleidervish, personal communication). The model by Baranauskas et al. (2010) implemented standard Hodgkin-Huxley equations (Hodgkin and Huxley 1952a,b; Hodgkin et al., 1952; Dayan and Abbott 2001) unless specified. Parameters used in the model by Baranauskas et al. (2010) were as follows.

Model morphology. Model morphology included soma (25 $\mu \mathrm{m}$ wide and $35 \mu \mathrm{m}$ long), apical dendrite ( $200 \mu \mathrm{m}$ long, tapering from 4 to $1 \mu \mathrm{m}$, with two $90-\mu \mathrm{m}$-long branches), and two basal dendrites (70 $\mu \mathrm{m}$ long, tapering from 2 to $0.5 \mu \mathrm{m})$. The axon consisted of a $10 \mu \mathrm{m}$ hillock, tapering from 4 to $1.2 \mu \mathrm{m}$, two AISs, each $20 \mu \mathrm{m}$ long and $1.2 \mu \mathrm{m}$ thick, followed by 10 myelinated sections (each $98 \mu \mathrm{m}$ long, $1.2 \mu \mathrm{m}$ thick) with nodes of Ranvier ( $2 \mu \mathrm{m}$ long, $1.1 \mu \mathrm{m}$ thick), and a non-myelinated terminal (100 $\mu \mathrm{m}$ long, tapering from 1.2 to $0.1 \mu \mathrm{m}$ ).

Passive properties. The passive properties included axial resistance $R_{\mathrm{ax}}=150 \Omega$-cm (standard value) or $R_{\mathrm{ax}}=300 \Omega$-cm in models 5 and 6 from Figure 3, leak conductance $G_{\text {Leak }}=0.33 \mathrm{pS} / \mu \mathrm{m}^{2}$, corresponding to input resistance of $51 \mathrm{M} \Omega$, leak current reversal potential set to $E_{\text {leak }}=$ $-80 \mathrm{mV}$, and membrane capacitance $C_{\mathrm{m}}=0.75 \mu \mathrm{F} / \mathrm{cm}^{2}$ everywhere except for the myelinated axon sections, in which it was $0.02 \mu \mathrm{F} / \mathrm{cm}^{2}$.

Sodium conductance. $\mathrm{gNa}$ was modeled using three types of channel kinetics: Hodgkin-Huxley type, threshold activation, and cooperative activation. For Hodgkin-Huxley-type kinetics, we used the script (Mainen et al., 1995; Mainen and Sejnowski, 1996) downloaded from the SenseLab ModelDB database (http://senselab.med.yale.edu/senselab/ modeldb), which implements standard equations (Hodgkin and Huxley, 1952a,b; Hodgkin et al., 1952; Dayan and Abbot, 2001). The activation and inactivation midpoint for sodium channels was -35 and $-65 \mathrm{mV}$, respectively, in all cell compartments except for the distal AIS, in which the activation midpoint was $-43 \mathrm{mV}$ whereas the inactivation $V_{1 / 2}$ was unchanged. Activation of sodium channels at more negative potential secured initiation of APs in the distal AIS (20-40 $\mu \mathrm{m}$ from the soma). $g \mathrm{Na}$ in the soma was $g \mathrm{Na}_{\text {soma }}=200 \mathrm{pS} / \mu \mathrm{m}^{2}$. In the AIS, $g \mathrm{Na}$ was 3,10 , 50 , or 100 times higher than in the soma, which is $g \mathrm{Na}_{\mathrm{AIS}}=600,2000$, 10,000 , and $20,000 \mathrm{pS} / \mu^{2}$; in the axon hillock, $g \mathrm{Na}$ was $50 \%$ of that in the AIS and in the Nodes of Ranvier, $g \mathrm{Na}_{\text {node }}=10,000 \mathrm{pS} / \mu \mathrm{m}^{2}$. Equilibrium potential for $\mathrm{Na}^{+}$ions, $E_{\mathrm{Na}}$, was set to $60 \mathrm{mV}$. In the HodgkinHuxley-type models designed to achieve a fast-onset dynamics of APs in the soma (Fig. 3, models 5 and 6), a combination of increased axial resistance $\left(R_{\mathrm{ax}}=300 \Omega-\mathrm{cm}\right)$ and extremely high density of sodium channels in the AIS $\left(10,000\right.$ or $\left.20,000 \mathrm{pS} / \mu \mathrm{m}^{2}\right)$ was used (for discussion of these parameters, see Baranauskas et al., 2010).

Model with a fraction of threshold channels (Fig. 3, models 8 and 9) had a moderate density of sodium channels in the AIS (10 times higher than in the soma), and $10 \%$ of sodium channels in the distal AIS had a modified kinetics that mimicked threshold activation. In the fraction of 


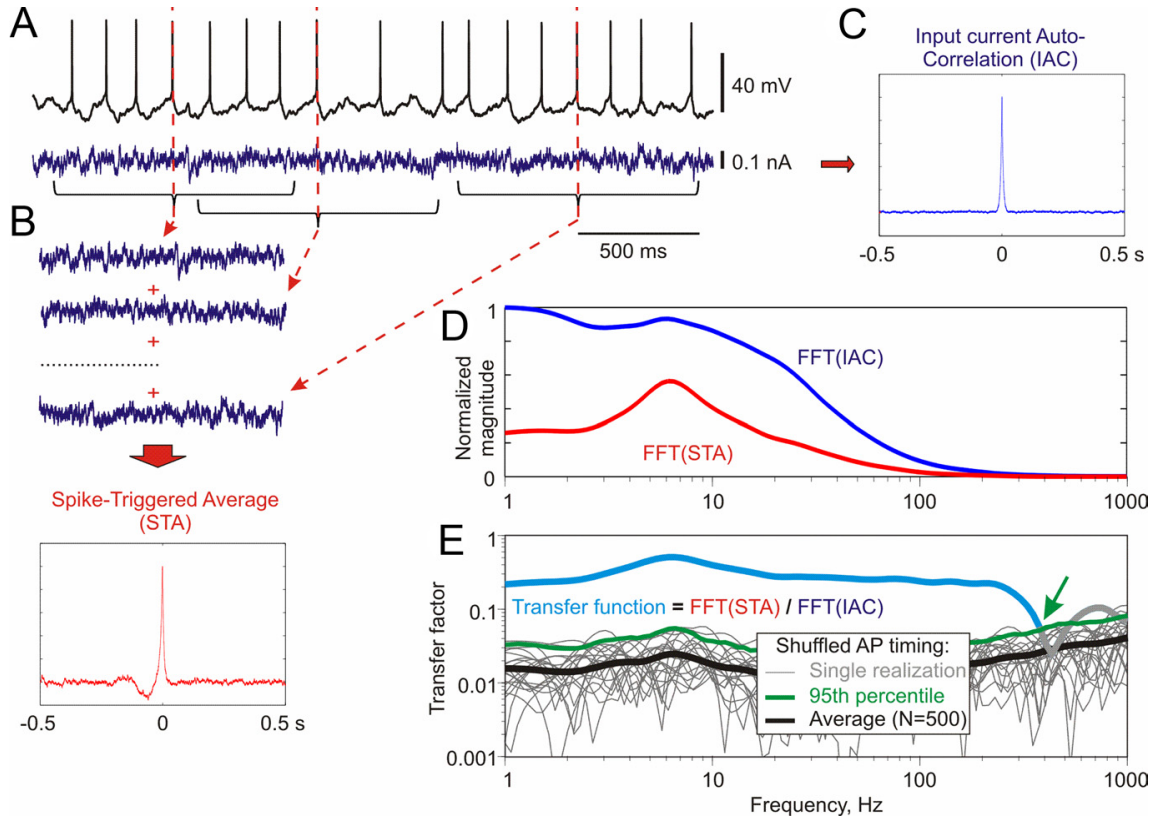

Figure 1. Calculation of frequency transfer function using noise injection. $\boldsymbol{A}$, Response of a neocortical neuron to injection of fluctuating current. $\boldsymbol{B}$, Spike-triggered average (STA) of the injected current. $\boldsymbol{C}$, Autocorrelation of the injected current (IAC). D, Fast Fourier transforms of the spike-triggered average FFT(STA) and of the input autocorrelation FFT(IAC). $\boldsymbol{E}$, Transfer function (cyan) calculated as the ratio of fast Fourier transforms of the spike-triggered average and the input autocorrelation. Method based on (Higgs and Spain, 2009). Gray lines show $n=20$ transfer functions calculated from the same recording but using shuffled AP timing. An average of $n=500$ transfer functions obtained with shuffled AP timing is shown as thick black line, and their 95 th percentile is shown as thick green line. Transfer function was considered significant when above the 95th percentile and cut at the intersection with 95th percentile (green arrow).

threshold channels, inactivation and subthreshold kinetics remained of Hodgkin-Huxley type, but when the membrane potential reached the threshold $(-70 \mathrm{mV})$, all available channels of this fraction open at once. This led to a very fast, step-like dynamics of AP onset at the initiation site (Fig. 3C, model 8, gray curves). All other parameters were as in the model with Hodgkin-Huxley-type channels.

Model with a fraction of cooperative channels (Fig. 3, model 7) had a moderate density of sodium channels in the AIS (10 times higher than in the soma). A fraction of sodium channels in the distal AIS (10\% of channels) expressed cooperative activation. In the fraction of cooperative channels, each channel is coupled to $K$ neighboring channels so that the opening of each neighbor increases the probability of the channel to open (Naundorf et al., 2006; Huang et al., 2012). The strength of coupling is proportional to the number of coupled channels $K$ and the coupling constant $J$. J has units of millivolts and defines the shift along the activation curve that would increase the open probability of an isolated channel by the same amount as opening of a coupled channel. In the simulations presented here, we used strong cooperativity, with $K J=3200 \mathrm{mV}$. Complete description of cooperative model of sodium channels and a MATLAB script of a single-compartment model is available in the study by Huang et al. (2012). All other parameters were as in the model with Hodgkin-Huxley-type channels.

Potassium conductance. Potassium conductance was implemented using the script of channel kinetics (Mainen et al., 1995; Mainen and Sejnowski, 1996) downloaded from SenseLab ModelDB database (http://senselab.med.yale.edu/senselab/modeldb) and modified as follows. The model script was rewritten for clarity and $n^{* *} 1$ changed to $n^{\star *} 4$. The activation midpoint was shifted by approximately $+6 \mathrm{mV}$ (to $-25 \mathrm{mV}$ ), which is closer to the original delayed rectifier current (Hodgkin and Huxley, 1952a,b; Hodgkin et al., 1952) and corresponds to the recent data on potassium current in the AIS (Kole et al., 2008). Peak current density was set to $I_{\mathrm{kv}}=400 \mathrm{pS} / \mu \mathrm{m}^{2}$ in the soma, $I_{\mathrm{kv}}=1000$ $\mathrm{pS} / \mu \mathrm{m}^{2}$ in the AIS, and $I_{\mathrm{kv}}=200 \mathrm{pS} / \mu \mathrm{m}^{2}$ in the dendrites. Slow delayed rectifier potassium current was modeled using the script from the model of Baranauskas et al. (2010). Peak current density was set to $I_{\mathrm{kd}}=600$
$\mathrm{pS} / \mu \mathrm{m}^{2}$ in the soma, $I_{\mathrm{kd}}=1200 \mathrm{pS} / \mu \mathrm{m}^{2}$ in the AIS, $I_{\mathrm{kd}}=1000 \mathrm{pS} / \mu \mathrm{m}^{2}$ in the nodes of Ranvier, and $I_{\mathrm{kd}}=300 \mathrm{pS} / \mu \mathrm{m}^{2}$ in the dendrites. The $\mathrm{K}^{+}$reversal potential was set to $E_{\mathrm{K}}^{+}=-85$ $\mathrm{mV}$.

All simulations were performed with $10 \mu$ s integration step at virtual temperature $32^{\circ} \mathrm{C}$.

Processing of membrane potential responses. Processing of the recorded and simulated membrane potential responses to injected current was done offline in MATLAB (MathWorks). Spikes were detected in membrane potential traces as crossings of zero voltage with positive slope. The timing of each spike $t_{j}$ was determined and saved in a spike train vector $s t(j)$. Onset dynamics of APs was quantified using onset rapidness (Naundorf et al., 2006) measured as a slope of the phase plot at $20 \mathrm{mV} / \mathrm{ms}$. Note that all electrophysiological recordings presented in this study were made from soma of neocortical neurons, and thus all parameters of APs in neocortical neurons were measured in the soma. In the multicompartmental models, we have measured AP parameters in both the soma, for comparison with electrophysiological data, and the axon at the site of AP initiation.

We used two methods to calculate frequency transfer function of neurons (or model neurons). In the first method, frequency transfer function was calculated from responses to injected fluctuating current using a modification of the method introduced recently by Higgs and Spain (2009). This method is illustrated in Figure 1 and consisted of the following steps. Using the fluctuating component of injected current $s(t)=\sigma_{\text {noise }} \eta(t)$ from Equation 1, we calculated input current autocorrelation $\left(c_{s s}\right)$ and spike-triggered average $\left(c_{s r}\right)$ :

$$
\begin{aligned}
& c_{\mathrm{ss}}(\tau)=\langle s(t) s(t+\tau)\rangle, \\
& c_{\mathrm{sr}}(\tau)=\langle s(t) r(t+\tau)\rangle,
\end{aligned}
$$

where $\tau$ is the time shift, and $r(t)$ is the postsynaptic spike train. $r(t)$ has the same length as $s(t)$, with spikes represented by 1 , and the remaining elements are 0 . To reduce the noise in Fourier transforms $C_{s r}$ of the spike-triggered average $c_{s r}$ and $C_{s s}$ of the input autocorrelation $c_{s s}$, we used frequency-dependent Gaussian filter $w(f, \tau)$. Filter $w(f, \tau)$ had a variable bandwidth defined by $\sigma_{w}=1 / f$ :

$$
w(f, \tau)=\exp \left(-\tau^{2} / 2 \sigma_{w}^{2}\right)=\exp \left(-\tau^{2} f^{2} / 2\right) .
$$

The Fourier transforms $C_{s r}$ and $C_{s s}$ were calculated for frequencies $f$ ranging from 1 to $1000 \mathrm{~Hz}$ with increments of 0.03 on a logarithmic scale:

$$
\begin{aligned}
& C_{\mathrm{sr}}(f)=\sum\left(c_{\mathrm{sr}}(\tau) w(f, \tau) e^{(-i 2 \pi f \tau)} d \tau\right), \\
& C_{\mathrm{ss}}(f)=\sum\left(c_{\mathrm{ss}}(\tau) w(f, \tau) e^{(-i 2 \pi f \tau)} d \tau\right) .
\end{aligned}
$$

The transfer function (TF) was calculated as a ratio of Fourier transforms:

$$
T F=\left|C_{\mathrm{sr}}(f)\right| /\left|C_{\mathrm{ss}}(f)\right| .
$$

The second method of assessing frequency transfer function used an established Fourier paradigm (Köndgen et al., 2008; Boucsein et al., 2009; Tchumatchenko et al., 2011). In this paradigm (Fig. 2), fluctuating current injected into neurons contained a weak sinusoid signal of frequency $f$ immersed in noise (Fig. 2A):

$$
I(t)=I_{0}+\sigma_{\text {noise }} \eta(t)+\varepsilon \sin (2 \pi f t),
$$




\section{A signal +
Noise

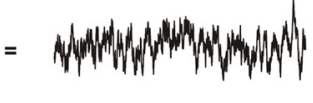
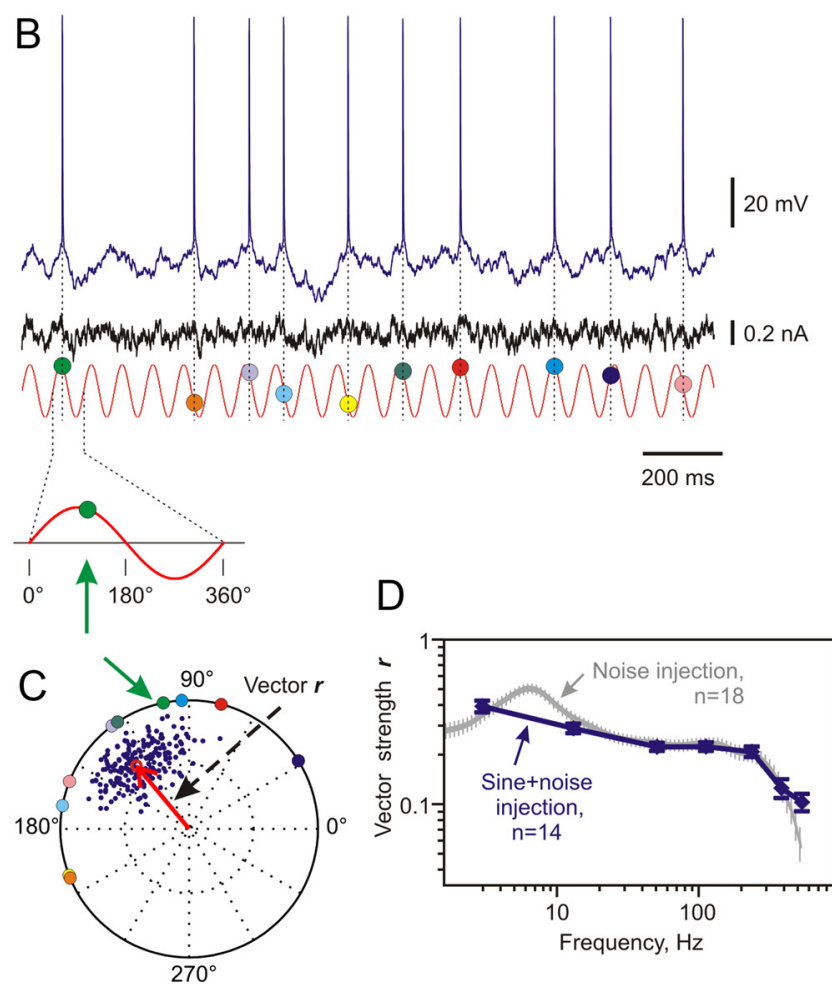

D

Figure 2. Calculation of frequency transfer function using responses to injection of sinewave signal immersed in fluctuating noise. $\boldsymbol{A}$, Current for injection consisting of a sine-wave signal of frequency $f$ immersed in fluctuating noise. $\boldsymbol{B}$, Membrane potential response of a neuron to current injection. Red trace shows sine-wave signal that was present in the injected current but at greatly expanded $y$-scale. Timing of each spike and its phase relative to the signal is shown by colored circles. $\boldsymbol{C}$, Calculation of the vector strength $\boldsymbol{r}$, which characterizes phaselocking of firing of the neurons to sine-wave signal of frequency $f$ and thus encoding of that frequency. Each spike is represented by a vector of unit length and a phase between 0 and $2 \pi$ defined as spike time modulo the stimulus period. Green circle at which green arrow is pointing shows the spike phase from the bottom in $\boldsymbol{B}$. Colored circles show phases of all spike vectors from $\boldsymbol{B}$. Sum of these vectors, normalized by the number of spikes, gives a vector $\boldsymbol{r}(\boldsymbol{r}=0.54)$, which length characterizes phase-locking of firing of the neurons to the stimulus and thus encoding of the stimulus frequency $f$. Dark-blue dots show results of bootstrapping, used to assess the variability of the vector length estimate $\boldsymbol{r}$. Each dot represents a mean vector calculated for random resamplings $(n=200)$ of $50 \%$ of experimentally measured spike times. Note that only 10 APs from a short period of stimulus presentation shown in $\boldsymbol{B}$ were used for the calculations, leading to high variability of bootstrapping results in this example. Results presented in $\boldsymbol{D}$ and in Results were obtained by processing much larger samples, $\sim 300$ to $\sim 3000$ spikes for each signal frequency per cell. $\boldsymbol{D}$, Vector strength $\boldsymbol{r}$ plotted against stimulus frequency provides an estimate of transfer function of neurons. Data from $n=14$ neurons (data from Fig. $5 B$ ). For comparison, transfer function measured with noise injections is shown (gray, data from Fig. 3B).

where $\varepsilon$ is signal amplitude with signal-to-noise ratio $\varepsilon /\left(\varepsilon+\sigma_{\text {noise }}\right)=$ 0.26 . Figure $2 B$ shows an example of responses of neurons to injected current. The sine-wave signal that was present in the injected current is shown at greatly expanded $y$-scale. For each spike, its phase shift relative to the signal was determined (Fig. $2 B$ ). Transfer function was estimated by the ability of neurons to phase-lock their firing to the signal. Phaselocking was characterized by the vector strength $r$, calculated as follows (Fig. 2C). Each spike was represented by a vector of unit length and the direction equal to the phase of the spikes $\varphi(0<\varphi<2 \pi)$ relative to the signal. Sum of these vectors, normalized by the number of spikes, gives a vector $\overline{\boldsymbol{r}}=\left(\sum_{j=1}^{N} \exp \left(i 2 \pi f t_{j}\right)\right) / \mathrm{N}$, where $t_{j}$ are the spike times and $N$ is the total number of spikes. The length of this vector $\boldsymbol{r}=a b s(\overline{\boldsymbol{r}})$ characterizes phase-locking of the firing of the neurons to the stimulus and thus encoding of the stimulus frequency $f$. Plotting vector strength $r$ against signal frequency $f$ provides an estimate of transfer function of neurons (Fig. 2D).

We used the first method of measuring transfer function with the use of injection of noise (experiments presented in Figs. 3, 4) because it allows to measure complete transfer function of a neuron within 20-25 min and thus measure transfer functions of the same neuron repeatedly, under different experimental conditions (as in experiments presented in Fig. 4). We used the second method that used injection of sine-wave signal immersed in noise (results in Fig. 5) because it has been used in previous studies (Köndgen et al., 2008; Boucsein et al., 2009; Tchumatchenko et al., 2011) and thus allows to compare the results. Because transfer functions measured using the two methods were similar (Fig. 2D), the results were pooled together for population analysis (Fig. 6).

Probability of detection of a step immersed in fluctuating noise current (Fig. 7) was calculated using a theoretical decoder that reports a change of the input when firing rate of a population of $n=1000$ (or $n=$ 3000) neurons exceeds the 95th percentile of pre-step firing rate. The probability of step detection as a function of time interval $T$ after the step (from 0.5 to $10 \mathrm{~ms}$ after step onset) was calculated as follows. The distribution of the number of spikes in a window of length $T$ after the step onset $D_{\text {post }}$ and the distribution of the number of spikes in a window of the same length before the step onset $D_{\text {pre }}$ were modeled as two independent binomial distributions with $N$ equal to the number of neurons ( $n=1000$ or $n=3000)$ and success probabilities $P_{\text {post }}$ (after step onset) and $P_{\text {pre }}$ (before step onset), respectively. The success probabilities $P_{\text {post }}$ and $P$ pre were estimated using responses to all steps in all recorded neurons as average probabilities of spikes:

(number of spikes in window $T$ )/(total number of current steps).

The theoretical detection probabilities were then computed as the probability that a binomial distribution $B\left(N, P_{\text {post }}\right)$ exceeds the $95 \%$ quantile of a binomial distribution $B\left(N, P_{\text {pre }}\right)$ :

$$
1-F_{B(N, P \text { post })}\left(F_{B(N, P \text { pre })}^{-1}(0.95)\right) \text {, }
$$

with $F_{B(N, P \text { post })}$ and $F^{-1}$ (N,Ppre) denoting the distribution and quantile function of a binomial random variable with parameters $N$ and $P$, respectively. Distributions and quantiles were computed using the MATLAB functions binoinv and binocdf.

\section{Results}

We first characterized encoding in neocortical neurons by measuring their frequency transfer function (Köndgen et al., 2008; Boucsein et al., 2009; Higgs and Spain, 2009; Tchumatchenko et al., 2011). We injected fluctuating current into the soma of layer $2 / 3$ pyramidal neurons of rat visual cortex in slices to evoke membrane potential fluctuations of $\sim 15-20 \mathrm{mV}$ peak to peak, 4.06 $\mathrm{mV} \mathrm{SD}$, which is typical range for in vivo activity (Azouz and Gray, 2000; Destexhe et al., 2003; Volgushev et al., 2006). A desired firing rate of $\sim 3-6 \mathrm{~Hz}$ (Figs. $1 A, 3 A$ ) was maintained by adding a direct current. Transfer function was calculated as the ratio of Fourier transforms of spike-triggered average and autocorrelation of the injected current, with frequency-dependent windowing used to improve the signal-to-noise ratio (Fig. 1). The significance of transfer functions was calculated using bootstrapping. For each neuron, we shuffled AP timing by circularly shifting spike timings in $r(t)$ by a random value using MATLAB function circshift. Transfer functions were then calculated using the shifted spikes. After repeating this procedure $n=500$ times, a 95th percentile of shuffled transfer functions was computed and used as confidence interval bound. Transfer functions were considered significant above this confidence interval bound (Fig. $1 E)$. Cumulative frequency transfer function ( $n=18$ cells) shows that the population firing of visual cortex neurons can encode 


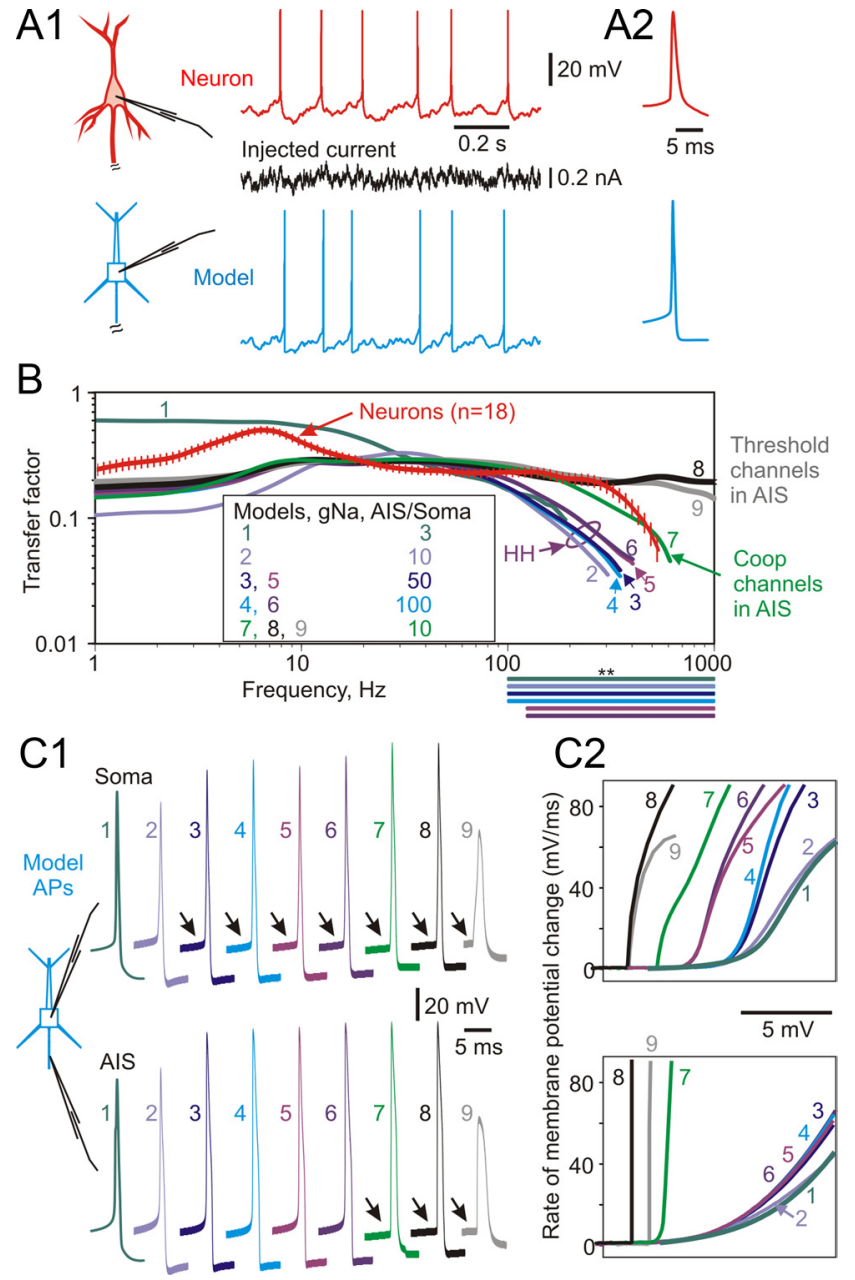

Figure 3. Frequency response functions of neocortical neurons and neuron models. A1, Response of a layer $2 / 3$ pyramidal neuron and a multicompartmental conductance-based neuron model to injection of the same fluctuating current in the soma. Both traces show membrane potential in the soma. $A \mathbf{2}$, APs in the neuron and in the model. $\boldsymbol{B}$, Frequency response functions measured in layer $2 / 3$ pyramidal neurons (red, $n=18$ neurons) and in neuron models. Models with Hodgkin-Huxley (HH)-type channels [1, from Fleidervish et al., 2010; 2-6, from Baranauskas et al., 2010, with $\mathrm{Na}^{+}$channel density in the AIS 10,50, and 100 times higher than in the soma; in models 5 and 6 , cytoplasmic resistance was increased ( $\left.R_{\mathrm{ax}}=300 \Omega-\mathrm{cm}\right)$ to achieve faster onset of AP in the soma; 7 , model with $10 \%$ of sodium channels in the AIS exhibiting cooperative activation; $\mathbf{8 , 9}$, models with $10 \%$ "threshold" sodium channels in the AlS; in models 7-9, other settings as in model 2; in model 9 , reduced driving force for sodium, $E_{\mathrm{Na}}=0$, and $g \mathrm{Na}$ by $\left.16 \%\right]$. In the threshold channel model, all available (non-inactivated) channels of this fraction open simultaneously when membrane potential reaches a threshold; inactivation and deinactivation dynamics as in Hodgkin-Huxley model. Bars below the plot show high-frequency ranges in which transfer factors in the recorded neurons were significantly higher $\left({ }^{* *} p<0.01\right)$ than in the color-coded model. C, Waveform of APs $(\mathbf{C})$ and their initial portion in phase plot $(\mathbf{C})$ in the soma and at the initiation site in the AIS in the models from $\boldsymbol{B}$, same number and color code. Phase plots in $\boldsymbol{C}$ are shifted along the $\boldsymbol{X}$-axis to facilitate comparison of onset dynamics.

frequencies up to $\sim 400 \mathrm{~Hz}$ (Fig. $3 B$, red curve). A cutoff frequency in the range of hundreds of hertz is compatible with a number of experimental measurements of the frequency response using noise injection in layer $2 / 3$ neurons from rat motor cortex (Higgs, Spain 2009), measurements of frequency transfer function in layer 5 neurons from somatosensory cortex obtained using responses to amplitude-modulated or variance-modulated sine-wave signals immersed in noise in vitro (Köndgen et al., 2008; Boucsein et al., 2009) and our previous measurements of frequency transfer function of visual cortex neurons from layer
$2 / 3$ in rat slices using sine-wave signals immersed in fluctuating noise in vitro and sine-wave injection in cat visual cortex cells in vivo (Tchumatchenko et al., 2011).

Next, we used the exact same paradigm to assess encoding properties in the multicompartmental models that reproduce spatial pattern of AP generation in neocortical neurons: initiation in distal portion of the AIS and propagation in both directions, down the axon and backpropagation to the soma and dendrites (Stuart and Sakmann, 1994; Stuart et al., 1997; Palmer and Stuart, 2006). We started with the recent model in which the distribution of sodium channels was calibrated to describe the dynamics of intracellular $\left[\mathrm{Na}^{+}\right]$during AP generation in neocortical pyramidal neurons (Fleidervish et al., 2010). This model exhibits poor encoding of fast frequencies. Transfer factors for frequencies above $\sim 90 \mathrm{~Hz}$ were significantly lower than measured in neurons (Fig. 3B, model $1, p<0.01$ for $f>93 \mathrm{~Hz}$ ). In this model, which was calibrated to account for dynamics of intracellular $\mathrm{Na}^{+}$concentration measured with fast optical imaging of fluorescent sodium dye, the density of sodium channels in the AIS was three times higher than in the soma (Fleidervish et al., 2010). However, the density of sodium channels in the AIS of neocortical neurons is a controversial issue. Other studies that used immunochemical labeling techniques (Lorincz and Nusser, 2008, 2010) or combination of electrophysiological recordings and modeling (Kole et al., 2008; Kole and Stuart, 2012) suggested higher density of sodium channels in the AIS, up to 50-fold of the somatic. A high density of sodium channels in the AIS has been also suggested to explain the fast-onset dynamics of somatic APs (Yu et al., 2008). Therefore, we next examined whether increasing the density of sodium channels in the AIS could improve encoding of high frequencies. We used a model (Baranauskas et al., 2010) with sodium channel density in the AIS set to 10, 50, and 100 times higher than in the soma, which corresponds to $g \mathrm{Na}$ of 2000 , 10,000 , and $20,000 \mathrm{pS} / \mu \mathrm{m}^{2}$ in the AIS. The dramatic increase of sodium channel density did not lead to an improvement of encoding of high frequencies in these models. Transfer functions of all three models did not express appreciable differences despite a 10 -fold difference in sodium current density at AP initiation site. In all three models, transfer factors for frequencies above $\sim 90 \mathrm{~Hz}$ were lower than measured in neurons (Fig. 3B, models $2-4$; $p<$ 0.01 for $f>93 \mathrm{~Hz}$ ). Increasing the density of sodium channels in the AIS changed only little the AP onset at the AIS initiation site. It remained gradual in accordance with Hodgkin-Huxley channel kinetics (Fig. 3C, bottom row). In the soma, however, AP onset dynamics changed with increasing density of $\mathrm{Na}^{+}$channels in the AIS. In the model with sodium channel density in the AIS 50 or 100 times higher than in the soma, onset dynamics of AP in the soma was sharp (Fig. 3C, models 3 and 4, arrows in the top) as suggested by the lateral current scenario (Yu et al., 2008). Previous work (Baranauskas et al., 2010) has demonstrated that this dissociation between the smooth onset of AP at the initiation site and a steeper onset in the soma becomes even more pronounced in the models that combine a very high density of sodium channels in the AIS (50 and 100 times of the somatic, 10,000 and $\left.20,000 \mathrm{pS} / \mu \mathrm{m}^{2}\right)$ and high cytoplasmic resistance $\left(R_{\mathrm{ax}}=300\right.$ $\Omega$-cm; Fig. 3, models 5 and 6). Despite a fast onset of somatic APs, encoding of high frequencies in these models remained clearly inferior to encoding in real neurons. Transfer factors for frequencies $>107 \mathrm{~Hz}$ were significantly lower in these models than in neurons (Fig. $3 B$ ). Thus, conductance-based models that reproduce distal initiation and backpropagation of APs but have slow, Hodgkin-Huxley type-onset dynamics of AP initiation in the AIS exhibit impaired encoding of high frequencies compared with 

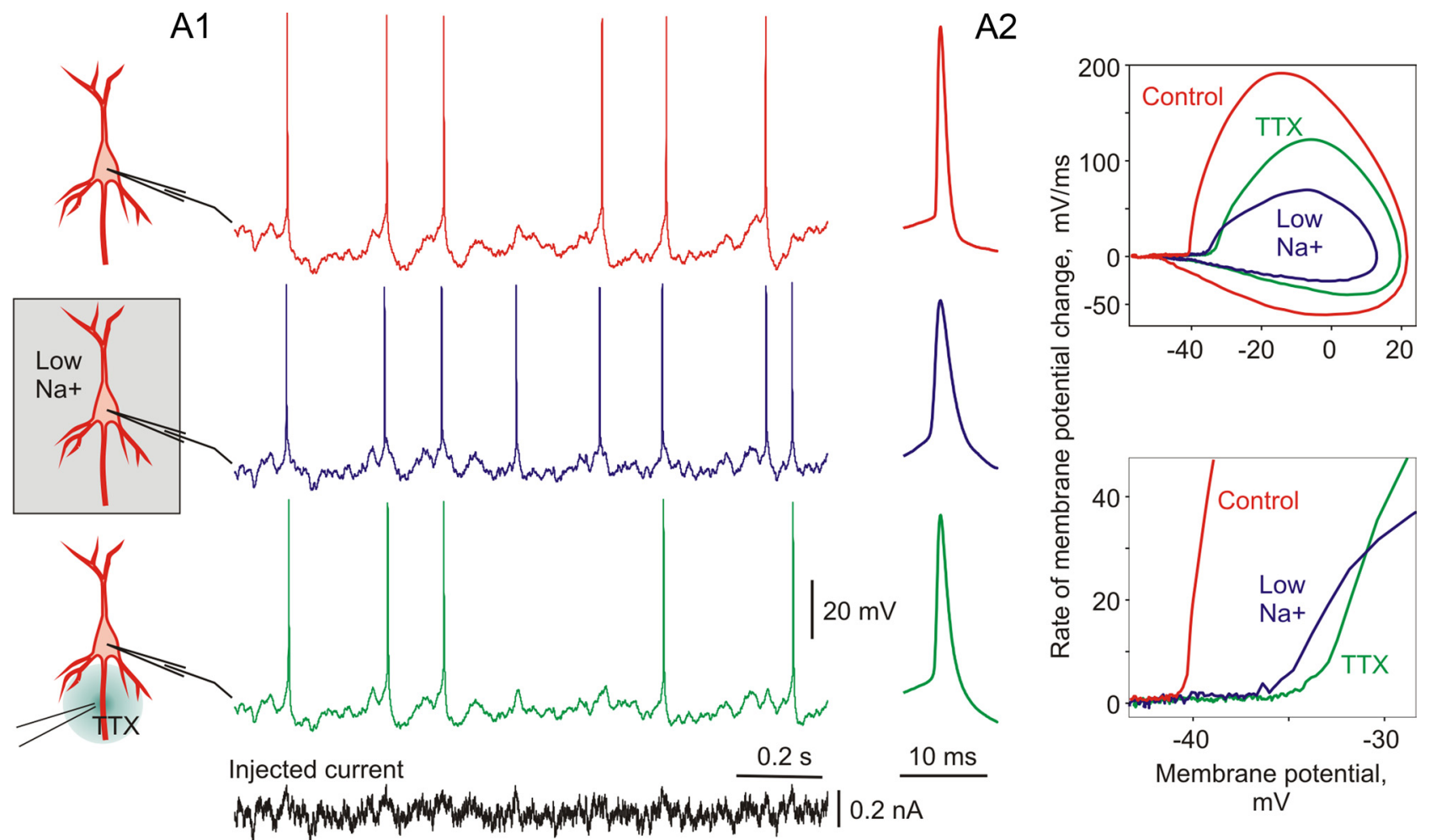

B
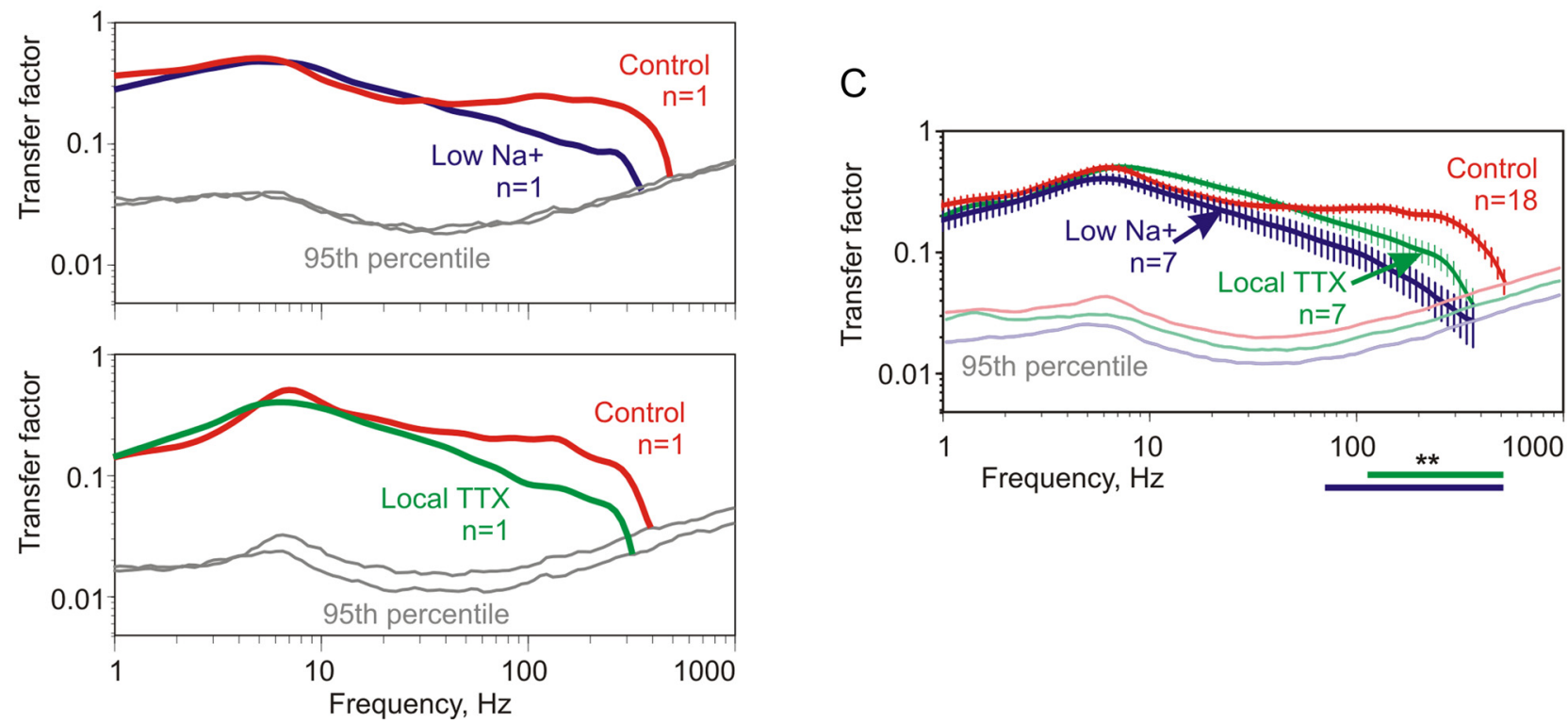

Figure 4. Impairment of encoding of high frequencies by manipulations that slow down AP onset dynamics. A1, Responses of neocortical neurons to injection of the same fluctuating current (bottom trace, black) in control conditions (red), in reduced extracellular [ $\left.\mathrm{Na}^{+}\right]$(blue), and during local application of TTX at AIS (green). All recordings are from the soma. A2, AP waveforms, phase plots, and zoom-in of initial portion of APs in phase plots illustrate slower onset of APs in low extracellular [ $\mathrm{Na}^{+}$] or during local TTX application relative to control. $\boldsymbol{B}$, Frequency-response functions of two neocortical neurons recorded in control conditions (red) and then either in low $\left[\mathrm{Na}^{+}\right]$(blue) or during local TTX application at the AIS (green). Transfer functions were cut at intersection with respective 95th percentile of $n=500$ transfer functions obtained with shuffled AP timings (gray). C, Averaged frequency-response functions measured in control conditions (red, $n=18 \mathrm{neurons),}$ in low $\left[\mathrm{Na}^{+}\right]$(blue, $n=7$ ), and during local TTX application at the AIS (green, $n=7$ ). Transfer functions were cut at respective 95th percentiles (pale color lines). Bars below the plot show high-frequency ranges in which encoding in low $\left[\mathrm{Na}^{+}\right]$or TTX experiments is significantly impaired $\left({ }^{* *} p<0.01\right)$ relative to control.

real neurons. Notably, encoding of high frequencies remained impaired also in the models tuned in such a way that lateral current invading the soma from the AIS produced fast onset of somatic APs (Yu et al., 2008; Baranauskas et al., 2010).

Could the impaired encoding of high frequencies in our models be attributable to low-pass-filtering properties of the AIS, so that high-frequency components of somatic membrane potential fluctuations did not reach the AP initiation site? To test for this possibility, we used a model with a moderate density of sodium channels in the AIS (10 times somatic, $2000 \mathrm{pS} / \mu^{2}{ }^{2}$ ) and modified a fraction $(10 \%)$ of sodium channels in the distal AIS to mimic threshold activation. In the fraction of threshold channels, inactivation and subthreshold kinetics remained of HodgkinHuxley type, but when the membrane potential reached the 


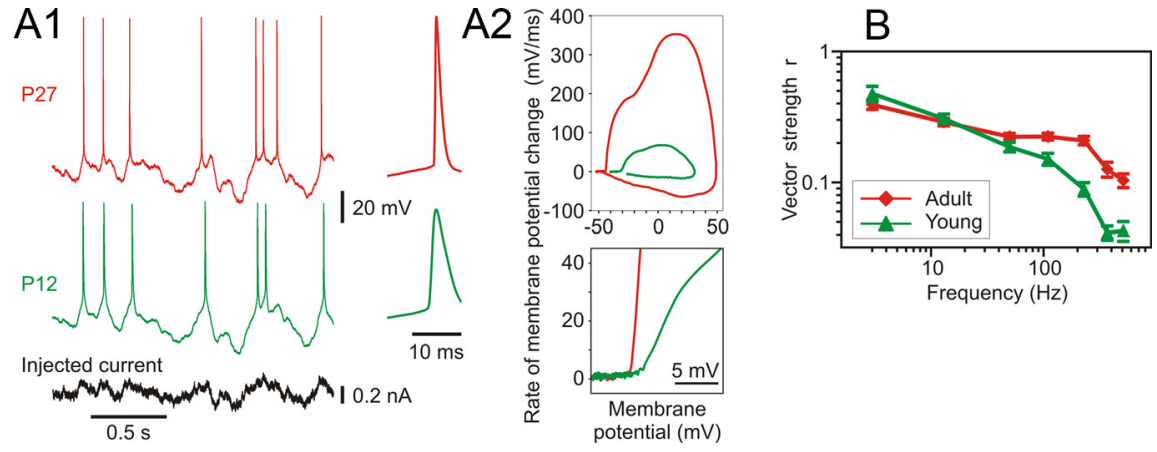

Figure 5. Neurons from young animals exhibit slow AP onset dynamics and impaired ability to encode high frequencies. A1, Responses of pyramidal neurons from adult (P27, red) and young (P12, green) animals to injection of the same fluctuating current (black). All recordings are from the soma. A2, AP waveforms, phase plots, and zoom-in of initial portion of APs in phase plots in neurons from $\boldsymbol{A} 1$, same color code. $\boldsymbol{B}$, Dependence of vector strength $\boldsymbol{r}$ on the signal frequency in neurons from young (green, $\mathrm{P} 9-\mathrm{P} 13, n=13$ neurons) and adult (red, P17-P30, $n=14$ ) animals. Note that, for high frequencies $>50 \mathrm{~Hz}$, adult neurons express significantly stronger phase locking to the stimulus than neurons from young animals.

threshold $(-70 \mathrm{mV})$, all available channels of this fraction open at once. This led to a very fast, step-like dynamics of AP onset at the initiation site and improved encoding of high frequencies dramatically (Fig. $3 B, C$, black curves, model 8). The frequency response of the model with a fraction of threshold channels at AP initiation site did not show cutoff up to the highest frequency tested $(1000 \mathrm{~Hz})$, which is well above the frequencies encoded by real neurons. Previous theoretical studies showed that such nondecrement transfer function is a characteristic signature of encoding in single-compartment threshold models with instantaneous AP initiation (Brunel et al., 2001; Fourcaud-Trocmé et al., 2003; Wei and Wolf, 2011). Notably, improved encoding of high frequencies survived, decreasing the virtual driving force for sodium $E_{\mathrm{Na}}$ from +60 to $0 \mathrm{mV}$ and reducing an overall density of sodium channels by $16 \%$ (Fig. $3 B$, dark gray curve). In this model, APs became smaller, broader, and had reduced maximal rate of rise but still exhibited sharp onset dynamics at the initiation site as a result of the threshold channels in AIS (Fig. 3C, model 9). Thus, low-pass filtering of the somatic signals introduced by passive properties of neurons, such as axial cytoplasmic resistance and membrane capacitance and resistance, cannot account for the impairment of encoding of high frequencies in the models with distal AP initiation described above. Moreover, small amplitudes and long duration of APs per se did not lead to impairment of encoding. Rather, the results indicate that onset dynamics of AP at initiation site, and thus kinetics of activation of sodium channels at the site of AP initiation, is crucial for encoding.

Previous work demonstrated that one possibility to capture fast-onset dynamics of APs in a single-compartment model is to introduce cooperative activation of sodium channels (Naundorf et al., 2006). Moreover, a single-compartment model with a fraction of cooperative channels exhibits an improved encoding of high frequencies (Huang et al., 2012). To test whether cooperativity can improve encoding in multicompartment model with distant AP initiation, we have introduced coupled gating in a small fraction $(10 \%)$ of sodium channels at the site of AP initiation in the distal AIS. The model had moderate channel density in the AIS, 10 times higher than in the soma, or $2000 \mathrm{pS} / \mu \mathrm{m}^{2}$. Encoding of high frequencies was significantly improved in this model. No significant difference was found between transfer factors for this model and recorded neurons (Fig. 3B, green, model 7). Moreover, transfer factors for frequencies $>50 \mathrm{~Hz}$ were significantly higher for the model with $10 \%$ of cooperative channels in the AIS (Fig. 3B, model 7) than in any model with Hodgkin-Huxley-type channels (Fig. 3B, models 1-6).

Thus, in multicompartment models with distal AP initiation, fast AP onset at the initiation site is crucial for the ability to encode high frequencies. With a gradual AP initiation, neither an increase of sodium channel density in the AIS nor an increase of the steepness of AP onset in the soma that was produced by the lateral current (Yu et al., 2008) lead to an appreciable improvement of encoding. Neurons encode high frequencies better than models with gradual Hodgkin-Huxley-type activation of sodium channels and slow AP onset at the site of initiation. This suggests that, at the site of AP initiation in neocortical neurons, a mechanism accelerating the activation of sodium channels and the onset of APs might be present. One possibility to achieve this could be coupled gating of a fraction of sodium channels at AP initiation site.

\section{Encoding is impaired in neurons with slowed down dynamics of AP onset}

APs in the soma of cortical neurons are characterized by very rapid, step-like onset dynamics (Naundorf et al., 2006; Yu et al., 2008). We have proposed that fast-onset dynamics of cortical AP generators can be attributable to cooperative gating of sodium channels and is beneficial for encoding of rapidly changing signals (Naundorf et al., 2006, 2007). We argued that, if cooperativity between sodium channels at the site of AP initiation is distance dependent, it should be reduced by manipulations that reduce effective density of sodium channels. Our previous results show, in agreement with this prediction, that decreasing effective density of sodium channels and sodium currents with low concentrations of TTX or partial substitution of $\mathrm{Na}^{+}$by choline in the extracellular solution lead to slow down of onset dynamics of APs recorded in the soma (Naundorf et al., 2006; Volgushev et al., 2006). Simulation results for multicompartment models presented in Figure 3 and previous theoretical analysis of encoding in single-compartment models (Fourcaud-Trocmé et al., 2003; Naundorf et al., 2005; Wei and Wolf, 2011; Huang et al., 2012) predict that slowing down the AP onset dynamics at the initiation site should lead to an impairment of encoding of high frequencies. To test this prediction, we made recordings in reduced extracellular $\left[\mathrm{Na}^{+}\right]$, with sodium partially substituted with choline. In reduced extracellular $\left[\mathrm{Na}^{+}\right]$, APs recorded in the soma of neocortical neurons had a lower amplitude $(38.3 \pm 28.1$ vs $70.1 \pm$ $29.6 \mathrm{mV}$ in control), were broader $(2.47 \pm 0.22 \mathrm{vs} 1.14 \pm 0.01$ $\mathrm{ms}$ ), had significantly slower onset dynamics (rapidness, $5.1 \pm$ 0.81 vs $20.4 \pm 1.56 \mathrm{~ms}^{-1}$ ), and, unlike in control, did not show a sharp kink at the onset (Fig. $4 A$, blue traces). Encoding of high frequencies was severely impaired in these recording conditions. The impaired encoding of high frequencies was clear in individual neurons in which transfer function was measured in both control conditions and low extracellular $\left[\mathrm{Na}^{+}\right]$(Fig. $4 B$, top) and in group data, in which transfer factors for $f>65 \mathrm{~Hz}$ were significantly lower than in control (Fig. 4C). These results provide the first experimental evidence for the theoretically predicted relationship between the AP onset dynamics and encoding of highfrequency inputs. 
Reducing extracellular $\left[\mathrm{Na}^{+}\right]$in the bath solution affected all sodium channels throughout all neuronal compartments. Simulation results from Figure 3 show that encoding of high frequencies is determined by onset dynamics of APs at the initiation site. To test specifically the role of sodium channels at AP initiation site, we measured encoding during local application of TTX to the AIS, in which APs are initiated in cortical neurons (Stuart and Sakmann, 1994; Stuart et al., 1997; Palmer and Stuart, 2006; Kole and Stuart, 2012). Local TTX application to AIS led to a reduction of the amplitude $(47.4 \pm 6.9$ vs $70.1 \pm 29.6 \mathrm{mV}$ in control), a slight increase of the width $(1.36 \pm 0.03$ vs $1.14 \pm$ $0.01 \mathrm{~ms}$ ), and slower onset dynamics (rapidness, $12.3 \pm 1.9$ vs $20.4 \pm 1.56 \mathrm{~ms}^{-1}$ ) of APs recorded in the soma (Fig. $4 A$, green traces). Impairment of encoding of high frequencies during local TTX application was clearly evident in individual neurons recorded in control and during local TTX application (Fig. $4 B$, bottom). In group data transfer factors for $f>\sim 80 \mathrm{~Hz}$ were markedly reduced relative to control (Fig. $4 B, C ; p<0.05$ for $f>83 \mathrm{~Hz} ; p<0.01$ for $f>105 \mathrm{~Hz}$ ). Thus, undisturbed operation of sodium channels in the AIS is crucial for achieving fast-onset dynamics of APs in the soma of neocortical neurons and for encoding of high frequencies.

How are the results of experiments with reduced extracellular $\left[\mathrm{Na}^{+}\right]$and local TTX application related to the simulation results? In the model, changing the density of sodium channels, specifically at the AP initiation site, by a factor of 5 (Fig. 3 , models 3 vs 2 and 5 vs 2 ) or 10 (Fig. 3 , models 4 vs 2 and 6 vs 2 ) did change the onset dynamics of APs in the soma in accordance with the invasion scenario. However, this manipulation had no appreciable effect on the encoding of high frequencies. In marked contrast to the simulation results, experimental manipulations leading to a decrease of the effective density of sodium channels in neurons in slices changed both the onset dynamics of somatic APs and encoding of high frequencies. This discrepancy between the experimental and modeling results suggests that the manipulations used in slice experiments led not only to a decreased sodium current because of decreased density of sodium channels but also must have disturbed mechanisms in the AIS (e.g., cooperativity of channels) that were responsible for fast initiation of APs and encoding of high frequencies. This interpretation is supported by the comparison of encoding in models implementing cooperative or threshold channels in the AIS with those with canonical, Hodgkin-Huxleytype AP initiation. Models with cooperative or threshold channels encoded high frequencies similar to or better than neurons. Removing the cooperative or threshold activation led to significant decrease of encoding of high frequencies (Fig. 3, models 7-9
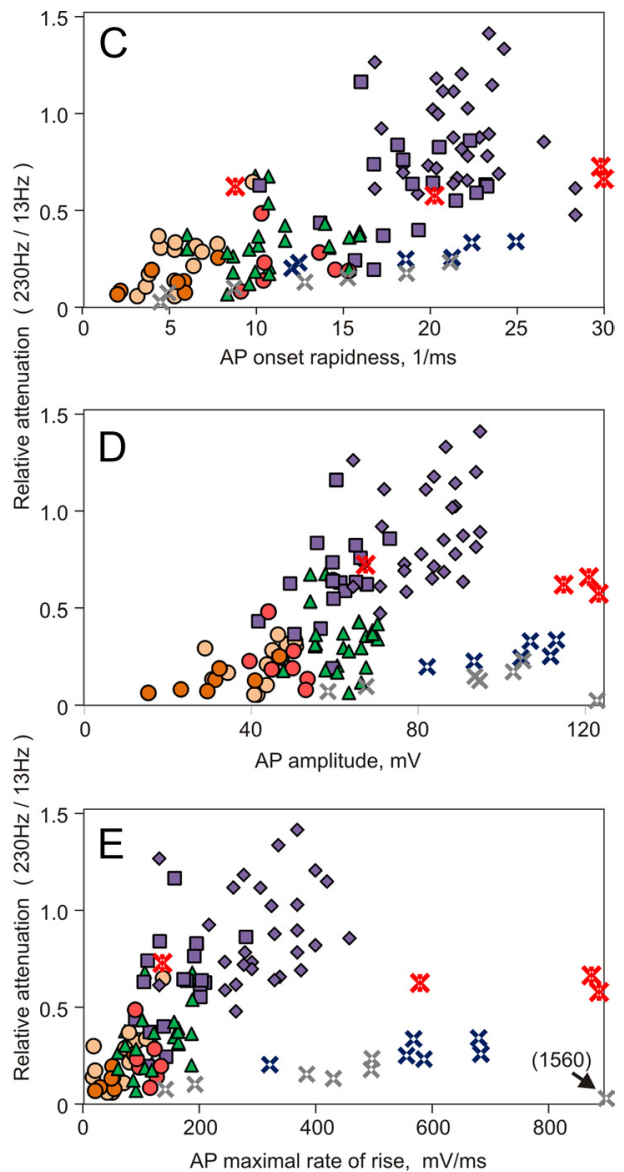

Figure 6. Dependence of the relative attenuation of encoding of high frequencies on properties of APs in neurons and models. Relative attenuation of encoding of high frequencies was quantified as the ratio of transfer factors (noise injection experiments, as (in experiments with sine-wave signals immersed in fluctuating noise, as in Figs. 4, 5) for encoding of $230 \mathrm{~Hz} / 13 \mathrm{~Hz}$. $\boldsymbol{A}-\boldsymbol{E}$, Dependence of the relative attenuation of encoding of high frequencies on age $(\boldsymbol{A})$, firing rate $(\boldsymbol{B}), A P$ ridness (C), AP amplitude (D), and AP maximal rate of rise $(\boldsymbol{E})$. In $\boldsymbol{A}-\boldsymbol{E}$, Data from young animals (P9-P13) are shown as green down of AP onset dynamics as circles, as indicated. Crosses show results of computer simulations. Blue crosses, Models with

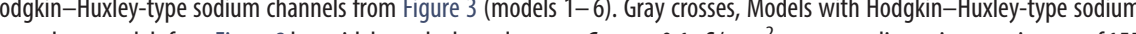
$\Omega$, and peak sodium current in all compartments reduced by $50 \%\left(\mathrm{gNa}_{\text {soma }}=100 \mathrm{pS} / \mu \mathrm{m}^{2}\right.$ instead of $200 \mathrm{pS} / \mu \mathrm{m}^{2}$, ratios in (he AIS), or very high gNa in the soma $1560 \mathrm{mV} / \mathrm{ms}$, but poor encoding of high frequencies). Red crosses, Models with a fraction of cooperative or threshold channels in the distal AIS (models 7-9 from Fig. 3). The model with a fraction of threshold channels but with $E_{\mathrm{Na}}=0 \mathrm{mV}$, and $g \mathrm{Na}$ reduced by $16 \%$ had lowest AP amplitude and rate of rise. The model with a fraction of cooperative channels but reduced sodium current (by $50 \%$ in all compartments) had lowest AP rapidness. In $\boldsymbol{C}-\boldsymbol{E}$, all parameters were measured using APs in the soma.

with a fraction of cooperative or threshold channels in the AIS vs models 1-6 with independent channels). These results indicate that a mechanism that accelerates activation of sodium channels and onset dynamics of APs and is dependent on undisturbed operation of sodium channels is present at the site of APs initiation in neocortical neurons.

To test whether encoding of high frequencies is also impaired in neurons exhibiting slow-onset somatic APs "naturally" without experimental manipulations, we compared encoding in neurons from adult and young animals. To compare data from adult neurons to previous studies (Köndgen et al., 2008; Boucsein et al., 2009; Tchumatchenko et al., 2011), we used an established Fourier paradigm to assess frequency transfer function (Köndgen et al., 2008; Boucsein et al., 2009; Tchumatchenko et al., 2011). Neurons were injected with currents consisting of weak sinusoidal signal of different frequencies immersed in fluctuating noise 

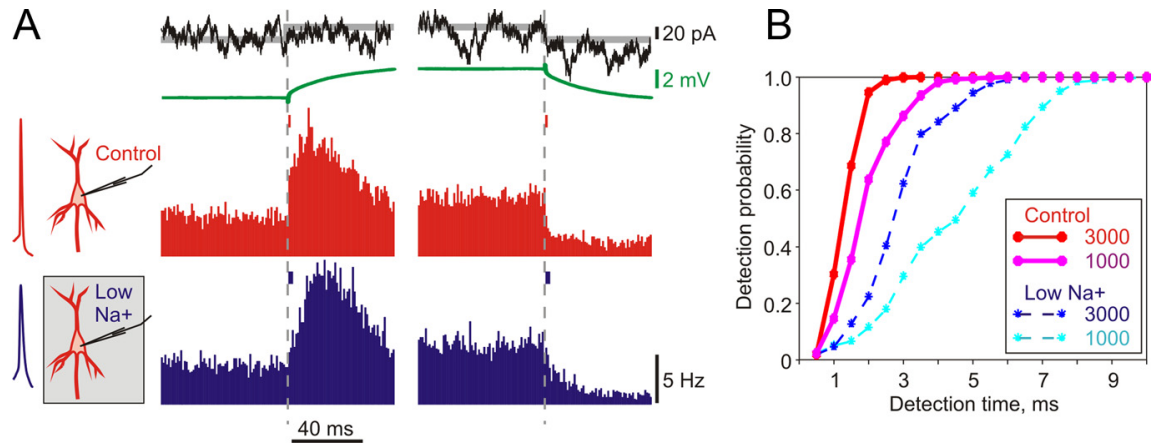

Figure 7. Artificial slowing down of AP onset decreases the speed of population firing rate response to current steps. $A$, Changes of population firing rate in response to the onset and offset of small current steps immersed in fluctuating current (top), in control conditions (red) and in the medium with reduced extracellular [ $\left.\mathrm{Na}^{+}\right]$, which leads to slower AP onset dynamics (blue; see Fig. 4). Green lines show membrane potential response to steps of the same amplitude but applied from subthreshold potential without noise. Example APs are shown on the left, and averaged AP width is shown above the histograms at step onset. Each peristimulus time histogram was calculated using $n=9100$ responses. $\boldsymbol{B}$, Probability of step detection versus time after step onset, for populations of 1000 or 3000 neurons. Note that detection time in low $\left[\mathrm{Na}^{+}\right]$is approximately twice longer than in control.

(Fig. 2A). Phase-locking of the firing of the neurons to the stimulus was characterized by the vector strength $\boldsymbol{r}$ (Fig. $2 B, C$ ). The two methods of measuring transfer function, using injection of noise or injection of sine-wave signal immersed in noise, gave very close results (Fig. 2D).

Onset dynamics of APs in the soma of neurons from young P9-P13 animals was slower than in adult P17-P30 neurons (rapidness, $11.2 \pm 2.5$ vs $20.4 \pm 1.56 \mathrm{~ms}^{-1}$; Fig. $5 A$ ), and encoding of frequencies $>50 \mathrm{~Hz}$ was significantly impaired (Fig. $5 B ; p<$ 0.003 for $110 \mathrm{~Hz}, p<0.0001$ for $230 \mathrm{~Hz}, p<0.0001$ for $370 \mathrm{~Hz}$, $p<0.001$ for $515 \mathrm{~Hz}$; Kruskal-Wallis test).

Figure 6 summarizes the above results. For each neuron, we measured attenuation of encoding of high frequencies as the ratio of transfer factors (experiments with noise injection; Figs. 1, 3, 4) or vector strengths (experiments with injection of sine-wave signals immersed in noise; Figs. 2, 5) for $230 \mathrm{~Hz} / 13 \mathrm{~Hz}$. The attenuation was strongly correlated with age for unmanipulated neurons (Fig. $6 A$, triangles, diamonds, and squares; $\rho=0.498$, Spearman's rank correlation, $n=61, p<0.001)$. Manipulations that slow down the onset of APs recorded in the soma impaired high-frequency encoding in adult neurons, bringing the attenuation in the range typical for young neurons with gradual AP onsets (Fig. 6A, $B$, circles) and conductance-based models with gradual AP initiation at distal portion of AIS (Fig. $6 B-E$, blue and gray crosses). Importantly, attenuation of high-frequency encoding could not be attributed to variability of the firing rate (Fig. $6 B$; $\rho=0.112 ; n=94, p=0.284$ ).

Attenuation of encoding of high frequencies was strongly correlated with several parameters of APs measured in the soma of neocortical neurons: AP onset rapidness $(\rho=0.789, p<0.001)$ but also with AP amplitude $(\rho=0.785, p<0.001)$ and maximal rate of rise $(\rho=0.813, p<0.001)$ (Fig. $6 C-E)$. All these parameters were inevitably affected by experimental manipulations used to slow down AP onset dynamics. To disentangle separate influence of each of these factors on encoding, we contrasted the results obtained in neurons against results of computer simulations. In conductance-based models with Hodgkin-Huxley-type sodium channels, AP amplitude and rate of rise in the soma varied dramatically depending on density and distribution of sodium channels, whereas onset dynamics of AP at initiation site remained slow (Fig. 3C). Notably, encoding of high frequencies was not improved in Hodgkin-Huxley-type models tuned to achieve high onset rapidness, amplitude, and rate of rise of so- matic APs (Fig. 6C-E, blue and gray crosses). Based on results of this study and on previous analysis of encoding in singlecompartment models (Fourcaud-Trocmé et al., 2003; Naundorf et al., 2005; Wei and Wolf, 2011; Huang et al., 2012), we attribute poor encoding of high frequencies to slow onset of APs at the initiation site. Models with slow onset of AP at the initiation site exhibited poor encoding of high frequencies even when tuned to achieve fast-onset dynamics of APs in the soma. In contrast, models with fast dynamics of AP onset at the initiation site (Fig. 6, red crosses, models with a fraction of cooperative or threshold channels in the AIS) encoded high frequencies comparable with or better than real neurons. Importance of AP onset dynamics at initiation site stands out especially clear when APs generated in models with Hodgkin-Huxley channels are compared with APs in threshold model with reduced density of sodium channels (by 16\%) and driving force for sodium $\left(E_{\mathrm{Na}}\right.$ $=0$ ). Somatic spikes in $E_{\mathrm{Na}}=0$ threshold model have lower amplitude, maximal rate of rise, and are broader than spikes generated by models with Hodgkin-Huxley channels but encode high frequencies clearly better. These results substantiate our conclusion that dynamics of AP onset at the site of initiation, rather than in other cell compartments, is crucial for encoding.

\section{Response speed of neuronal populations depends on onset dynamics of APs}

In a linear system, response timescale (response speed) is related to the cutoff frequency $f_{c}$ as $1 /\left(2 \pi f_{c}\right)$. The cutoff frequencies in the range of $\sim 300-400 \mathrm{~Hz}$, measured for populations of cortical neurons (Figs. 3B, 5B), imply that cortical ensembles can respond to sudden changes of the input on a submillisecond scale [1/ $(2 \pi 300 \mathrm{~Hz})<1 \mathrm{~ms}]$. Indeed, populations of cortical neurons can change their firing rate in response to small, 20 pA steps immersed in fluctuating noise extremely fast, on a millisecond timescale (Fig. 7A, red histograms, consistent with Tchumatchenko et al., 2011). However, when the onset of APs (as recorded in the soma) was modified to gradual by decreasing extracellular $\left[\mathrm{Na}^{+}\right]$, the population response lost its instantaneous component, but became slow, with dynamics comparable with the membrane time constant (Fig. 7A, blue histograms). To assess the impact of this change of the AP onset dynamics on the speed of computations in neuronal networks, we calculated how fast a change in population firing rate can be detected by a theoretical decoder. Theoretical decoder reports a change, when population firing rate falls outside $95 \%$ confidence boundary of pre-step distribution (Tchumatchenko et al., 2011). Time of step detection by the decoder was more than doubled in experiments with low $\left[\mathrm{Na}^{+}\right]$compared with control. For detection probability 0.9 , it increased from 1.8 to $4.5 \mathrm{~ms}$ for a population of $n=3000$ neurons and from 3.2 to $7.0 \mathrm{~ms}$ for a population of $n=1000$ neurons (Fig. $7 B$ ). This implies that a three-layer network consisting of neurons with rapid AP onset dynamics, as observed in cortical neurons, can process signals $\sim 10$ times faster than networks composed of neurons with gradual, Hodgkin-Huxleytype initiation of APs. Thus, an apparently minor modification of AP onset dynamics has dramatic consequences for the speed of neuronal computations. 


\section{Discussion}

The results of the present study show for the first time that encoding of fast changing signals and the speed of communication between ensembles of spiking cortical neurons is fundamentally modified by experimental manipulations that affect apparently minor details of AP initiation dynamics. Evidence obtained in both the frequency and time domain demonstrates that neurons with fast AP onsets can encode high frequencies and respond quickly (within 1-2 ms) to subtle input changes. However, this ability is impaired and response speed is decreased when AP onsets are slowed down by experimental manipulations. Exactly this behavior has been predicted by previous theoretical analyses of point models with modifiable dynamics of AP initiation (Fourcaud-Trocmé et al., 2003; Naundorf et al., 2005; Wei and Wolf, 2011). Our simulation results extend this prediction to the case of distal AP initiation, typical for cortical neurons (Stuart and Sakmann, 1994; Stuart et al., 1997; Palmer and Stuart, 2006; Kole and Stuart, 2012). We further show that only models with fast AP onset at the initiation site, achieved, for example, by introducing cooperative gating or threshold activation to a portion of sodium channels in the AIS, exhibit encoding of high frequencies compatible with that in real neurons. On the contrary, models with slow AP onset at the initiation site cannot capture encoding properties of cortical neurons, even when tuned to produce fast onset of somatic AP by the invasion scenario (Yu et al., 2008).

\section{AP onset dynamics at initiation site determines the encoding}

Several lines of evidence identify AP onset dynamics at the initiation site as a crucial factor determining encoding in populations of neocortical neurons. Model neurons were able to encode highfrequency signals only if APs had fast onset at the initiation site. This could be achieved by introducing cooperative gating or threshold activation to a fraction (10\%) of sodium channels at the AP initiation site. Notably, encoding of high frequencies changed little in model experiments with reduced driving force and peak conductance for $\mathrm{Na}^{+}$, in which APs had reduced amplitude, reduced rate of rise, and increased width but the same fast initiation dynamics produced by the fraction of threshold channels in the AIS. However, encoding of high frequencies was impaired in the models with a slow AP onset at the initiation site, produced by Hodgkin-Huxley-type $\mathrm{Na}^{+}$channels. In such models, encoding was not improved even when they were tuned to produce a rapid onset of somatic APs attributable to the invasion of current from AIS in the soma (Yu et al., 2008), and the density of Hodgkin-Huxley-type $\mathrm{Na}^{+}$channels in the AIS was changed by an order of magnitude (10 and 100 times the somatic, or from 2000 to $20,000 \mathrm{pS} / \mathrm{\mu m}^{2}$ ). The onset dynamics of AP at the initiation site remained gradual in these models, and encoding of high frequencies was not improved. Thus, AP onset at the initiation site, but neither the size of the invasion component nor its effect on AP kinetics in the soma, determines the encoding.

Neocortical neurons in which APs in the soma exhibit fastonset dynamics were able to encode high-frequency signals. This ability was impaired when AP onset dynamics was slowed down by experimental manipulations, such as reduced extracellular $\left[\mathrm{Na}^{+}\right]$, or local application of TTX to the AIS. Because simulation results discussed above show that AP onset at the initiation site determines the encoding of high frequencies, we suggest that, also in slice experiments, the disturbance of the operation of sodium channels in the AIS was the reason for the slow AP onset and impaired encoding.

\section{Sodium channels in the AIS are crucial for rapid AP onset and encoding}

Impairment of encoding during local application of TTX to the AIS stresses the importance of undisturbed operation of sodium channels in the AIS for encoding of high-frequency signals by neocortical neurons. Notably, although in neocortical neurons APs are initiated in the distal AIS (Stuart and Sakmann, 1994; Stuart et al., 1997; Palmer and Stuart, 2006; Kole and Stuart, 2012), encoding of high frequencies in slice experiments has been well predicted by the onset rapidness of APs recorded in the soma. Because encoding of fast changing signals critically depends on onset dynamics of AP at the initiation site, these results indicate that the onset of APs recorded in the soma reflects the dynamics of AP initiation in the AIS. Recent computational analysis of determinants of rapid-onset dynamics of somatic APs (Baranauskas et al., 2010) supports this conclusion.

Ability of neocortical neurons to encode high-frequency signals implies that AP onset should be fast at the initiation site. The proposal by Yu et al. (2008) that the sharpness of AP onset in the soma is an epiphenomenon produced solely by backpropagation of an AP that has a slow onset at the initiation site but becomes fast while invading the soma fails to explain the ability of neurons to encode high-frequency information, as well as the impairment of this ability by experimental manipulations that slow down the AP onset dynamics. Rather, fast onset of APs in the soma might reflect an intrinsic property of cortical AP generators (Naundorf et al., 2006, 2007).

Notion of a specific mechanism in the AIS of neocortical neurons that underlies fast initiation of APs is further supported by the discrepancy between changes of encoding induced by manipulations to sodium currents in slice experiments and in the models. In slice experiments, marked decrease of the ability of neurons to encode high frequencies was observed in reduced extracellular $\left[\mathrm{Na}^{+}\right]$or during local application of TTX at the AIS. If the only effect of these manipulations was a reduction of APrelated sodium currents attributable to reduced number of operating channels, similar effects on encoding would be expected when $g \mathrm{Na}$ is changed in the models. However, in the models, 5- or 10 -fold change of the peak $\mathrm{gNa}$ had little effect on encoding of high frequencies. At the same time, introducing a mechanism leading to fast onset of AP at the initiation site, such as threshold or cooperative activation of a fraction of $\mathrm{Na}^{+}$channels in the AIS, had dramatic effects on encoding of high frequencies, comparable in magnitude with those observed experimentally. These results suggest that reduced extracellular $\left[\mathrm{Na}^{+}\right]$or local application of TTX in slice experiments not only reduced $\mathrm{Na}^{+}$ currents but also disturbed mechanisms in the AIS that were responsible for fast AP initiation. One possible mechanism that was suggested to explain fast AP initiation is cooperative activation of a fraction of sodium channels at the site of AP initiation (Naundorf et al., 2006, 2007; Huang et al., 2012). Indeed, cooperative gating of a fraction of sodium channels improves encoding of high frequencies in both a singlecompartment model (Huang et al., 2012) and models with distant AP initiation (present results).

Cooperative gating has been reported for a wide variety of channels, such as $\mathrm{Na}^{+}$channels in myocytes (Undrovinas et al., 1992), $\mathrm{K}^{+}$channels (Molina et al., 2006), $\mathrm{Ca}^{2+}$ channels (Marx et al., 1998, 2001), and neurotransmitter-gated channels (Schindler 1984; Keleshian et al., 2000). Coupled gating can be achieved by direct protein-protein interactions, as in the case of ryanodine R2 receptors (Marx et al., 2001). Another intriguing possibility could be a positive feedback on sodium channel gating via an 
increase of intracellular $\left[\mathrm{Na}^{+}\right]$associated with AP initiation. Although no direct evidence for $\mathrm{Na}^{+}$dependence of gating of sodium channels was reported so far, existence of a sensor for intracellular $\mathrm{Na}^{+}$that regulates the gating of potassium channels (Dryer, 1994; Franceschetti et al., 2003; Bhattacharjee and Kaczmarek, 2005; $\mathrm{K}_{\mathrm{Na}}$ channels) makes this possibility worth considering. Although the specific biophysical mechanisms involved remain to be discovered, it is tempting to speculate that elaborated molecular architecture of the AIS (Inda et al., 2006; Lorincz and Nusser, 2008, 2010; Rasband 2010; Kole and Stuart, 2012) is essential for achieving rapid-onset dynamics of APs in neocortical neurons.

\section{Functional significance of fast AP initiation}

Fast AP initiation brings about several advantages for computations in multilayer networks with abundant feedforward and feedback connections. Ensembles of neurons equipped with mechanisms supporting fast AP initiation can detect an input change and report it to the next level faster than neurons in which initiation of APs is gradual. This allows for shortening the total time that is needed for signal processing in multilayer networks and/or increasing the number of iterations and number of ensembles that can contribute to the final result within a given time. Furthermore, with fast AP initiation, the same processing speed can be achieved with significantly smaller ensembles. In the example shown in Figure 7, a population of $n=1000$ neurons with fast AP onsets can detect a subtle step-like change of the input faster than a population of $n=3000$ neurons with slower AP onsets. This allows for tremendous decrease in the number of elements and connections and thus substantial reduction of energetic cost of neuronal computations. Increasing response speed and decreasing energetic costs are vital and highly desirable features for performing rapid computations in recurrent intracortical circuits, propagation of information through cortical area hierarchy, and the impact of top-down modulation within such hierarchies.

\section{References}

Azouz R, Gray CM (2000) Dynamic spike threshold reveals a mechanism for synaptic coincidence detection in cortical neurons in vivo. Proc Natl Acad Sci U S A 97:8110-8115. CrossRef Medline

Baranauskas G, Martina M (2006) Sodium currents activate without a Hodgkin-and-Huxley-type delay in central mammalian neurons. J Neurosci 26:671-684. CrossRef Medline

Baranauskas G, Mukovskiy A, Wolf F, Volgushev M (2010) The determinants of the onset dynamics of action potentials in a computational model. Neuroscience 167:1070-1090. CrossRef Medline

Bhattacharjee A, Kaczmarek LK (2005) For $\mathrm{K}^{+}$channels, $\mathrm{Na}^{+}$is the new $\mathrm{Ca}^{2+}$. Trends Neurosci 28:422-428. CrossRef Medline

Boucsein C, Tetzlaff T, Meier R, Aertsen A, Naundorf B (2009) Dynamical response properties of neocortical neuron ensembles: Multiplicative versus additive noise. J Neurosci 29:1006-1010. CrossRef Medline

Brunel N, Chance FS, Fourcaud N, Abbott LF (2001) Effects of synaptic noise and filtering on the frequency response of spiking neurons. Phys Rev Lett 86:2186-2189. CrossRef Medline

Carnevale NT, Hines ML (2006) The NEURON book. Cambridge, UK: Cambridge UP.

Dayan P, Abbott LF (2001) Theoretical neuroscience. Cambridge, MA: Massachusetts Institute of Technology.

Destexhe A, Rudolph M, Paré D (2003) The high-conductance state of neocortical neurons in vivo. Nat Rev Neurosci [Erratum (2003) 4:1019] 4:739-751. CrossRef Medline

Dryer SE (1994) $\mathrm{Na}^{+}$-activated $\mathrm{K}^{+}$channels: a new family of large conductance ion channels. Trends Neurosci 17:155-160. CrossRef Medline

Engel D, Jonas P (2005) Presynaptic action potential amplification by voltage-gated $\mathrm{Na}$ channels in hippocampal mossy fiber boutons. Neuron 45:405-417. CrossRef Medline

Fleidervish IA, Lasser-Ross N, Gutnick MJ, Ross WN (2010) $\mathrm{Na}^{+}$imaging reveals little difference in action potential-evoked $\mathrm{Na}^{+}$influx between axon and soma. Nat Neurosci 13:852-860. CrossRef Medline

Fourcaud-Trocmé N, Hansel D, van Vreeswijk C, Brunel N (2003) How spike generation mechanisms determine the neuronal response to fluctuating inputs. J Neurosci 23:11628-11640. Medline

Franceschetti S, Lavazza T, Curia G, Aracri P, Panzica F, Sancini G, Avanzini G, Magistretti J (2003) $\mathrm{Na}^{+}$-activated $\mathrm{K}^{+}$current contributes to postexcitatory hyperpolarization in neocortical intrinsically bursting neurons. J Neurophysiol 89:2101-2111. CrossRef Medline

Higgs MH, Spain WJ (2009) Conditional bursting enhances resonant firing in neocortical layer 2-3 pyramidal neurons. J Neurosci 29:1285-1299. CrossRef Medline

Hines ML, Morse T, Migliore M, Carnevale NT, Shepherd GM (2004) Model DB: a database to support computational neuroscience. J Comput Neurosci 17:7-11. CrossRef Medline

Hodgkin AL, Huxley AF (1952a) Currents carried by sodium and potassium ions through the membrane of the giant axon of Loligo. J Physiol 116:449472. Medline

Hodgkin AL, Huxley AF (1952b) A quantitative description of membrane current and its application to conduction and excitation in nerve. J Physiol 117:500-544. Medline

Hodgkin AL, Huxley AF, Katz B (1952) Measurement of current-voltage relations in the membrane of the giant axon of Loligo. J Physiol 116:424448. Medline

Huang M, Volgushev M, Wolf F (2012) A small fraction of strongly cooperative sodium channels boosts neuronal encoding of high frequencies. PLoS One 7:e37629. CrossRef Medline

Inda MC, DeFelipe J, Muñoz A (2006) Voltage-gated ion channels in the axon initial segment of human cortical pyramidal cells and their relationship with chandelier cells. Proc Natl Acad Sci U S A 103:2920-2925. CrossRef Medline

Keleshian AM, Edelson RO, Liu GJ, Madsen B (2000) Evidence for cooperativity between nicotinic acetylcholine receptors in patch clamp records. Biophys J 78:1-12. CrossRef Medline

Knight BW (1972) Dynamics of encoding in a population of neurons. J Gen Physiol 59:734-766. Medline

Kole MH, Stuart GJ (2012) Signal processing in the axon initial segment. Neuron 73:235-247. CrossRef Medline

Kole MH, Ilschner SU, Kampa BM, Williams SR, Ruben PC, Stuart GJ (2008) Action potential generation requires a high sodium channel density in the axon initial segment. Nat Neurosci 11:178-186. CrossRef Medline

Köndgen H, Geisler C, Fusi S, Wang XJ, Lüscher HR, Giugliano M (2008) The dynamical response properties of neocortical neurons to temporally modulated noisy inputs in vitro. Cereb Cortex 18:2086-2097. CrossRef Medline

Lorincz A, Nusser Z (2008) Cell-type-dependent molecular composition of the axon initial segment. J Neurosci 28:14329-14340. Medline

Lorincz A, Nusser Z (2010) Molecular identity of dendritic voltage-gated sodium channels. Science 328:906-909. CrossRef Medline

Mainen ZF, Sejnowski TJ (1996) Influence of dendritic structure on firing pattern in model neocortical neurons. Nature 382:363-366. CrossRef Medline

Mainen ZF, Joerges J, Huguenard JR, Sejnowski TJ (1995) A model of spike initiation in neocortical pyramidal neurons. Neuron 15:1427-1439. CrossRef Medline

Marx SO, Ondrias K, Marks AR (1998) Coupled gating between individual skeletal muscle $\mathrm{Ca}^{2+}$ release channels (ryanodine receptors). Science 281: 818-821. CrossRef Medline

Marx SO, Gaburjakova J, Gaburjakova M, Henrikson C, Ondrias K, Marks AR (2001) Coupled gating between cardiac calcium release channels (ryanodine receptors). Circ Res 88:1151-1158. CrossRef Medline

Molina ML, Barrera FN, Fernández AM, Poveda JA, Renart ML, Encinar JA, Riquelme G, González-Ros JM (2006) Clustering and coupled gating modulate the activity in KcsA, a potassium channel model. J Biol Chem 281:18837-18848. CrossRef Medline

Naundorf B, Geisel T, Wolf F (2005) Action potential onset dynamics and the response speed of neuronal populations. J Comput Neurosci 18:297309. CrossRef Medline

Naundorf B, Wolf F, Volgushev M (2006) Unique features of action potential initiation in cortical neurons. Nature 440:1060-1063. CrossRef Medline 
Naundorf B, Wolf F, Volgushev M (2007) Hodgkin and Huxley modelstill standing? (Reply) Nature 445:E2-E3. CrossRef

Palmer LM, Stuart GJ (2006) Site of action potential initiation in layer 5 pyramidal neurons. J Neurosci 26:1854-1863. CrossRef Medline

Paxinos G, Watson C (1997) The rat brain in stereotaxic coordinates. San Diego: Academic.

Rasband MN (2010) The axon initial segment and maintenance of neuronal polarity. Nat Rev Neurosci 11:552-562. CrossRef Medline

Schindler H (1984) Cooperativity between acetylcholine-receptor channels. Prog Clin Biol Res 164:291-294. Medline

Stuart GJ, Sakmann B (1994) Active propagation of somatic action potentials in to neocortical pyramidal cell dendrites. Nature 367:69-72. CrossRef Medline

Stuart G, Spruston N, Sakmann B, Häusser M (1997) Action potential initiation and backpropagation in neurons of the mammalian CNS. Trends Neurosci 20:125-131. CrossRef Medline

Tchumatchenko T, Malyshev A, Wolf F, Volgushev M (2011) Ultrafast population encoding by cortical neurons. J Neurosci 31:12171-12179. CrossRef Medline
Tuckwell H (1998) Introduction to theoretical neurobiology. Cambridge, UK: Cambridge UP.

Undrovinas AI, Fleidervish IA, Makielski JC (1992) Inward sodium current at resting potentials in single cardiac myocytes induced by the ischemic metabolite lysophosphatidylcholine. Circ Res 71:1231-1241. CrossRef Medline

Volgushev M, Vidyasagar T, Chistiakova M, Yousef T, Eysel UT (2000) Membrane potential and spike generation in rat visual cortical slices during reversible cooling. J Physiology [Erratum (2000) 528:669] 522: 59-76. CrossRef Medline

Volgushev M, Chauvette S, Mukovski M, Timofeev I (2006) Precise longrange synchronization of activity and silence in neocortical neurons during slow-wave oscillations. J Neurosci 26:5665-5672. CrossRef Medline

Wei W, Wolf F (2011) Spike onset dynamics and response speed in neuronal populations. Phys Rev Lett 106:088102. CrossRef Medline

Yu Y, Shu Y, McCormick DA (2008) Cortical action potential backpropagation explains spike threshold variability and rapid-onset kinetics. J Neurosci 28:7260-7272. CrossRef Medline 